\title{
Crosslinking Amine-Modified Silica Aerogels with Epoxies: Mechanically Strong Lightweight Porous Materials
}

Mary Ann B. Meador ${ }^{\dagger}$,Eve F. Fabrizio ${ }^{\ddagger}$ Faysal Ilhan ${ }^{\ddagger}$, Amala Dass ${ }^{\S}$, Guohui Zhang ${ }^{\S}$, Plousia Vassilaras $^{\dagger}$, J. Chris Johnston ${ }^{\dagger}$ and Nicholas Leventis ${ }^{*}{ }^{*}$

†NASA Glenn Research Center, 21000 Brookpark Rd., Cleveland OH 44135;

${ }^{\S}$ University of Missouri-Rolla, Rolla, Missouri 65409; and, ${ }^{*}$ Ohio Aerospace Institute, 22800 Cedar Point Rd., Cleveland OH 44142

\section{Supporting Information}

Appendix I: Plot of experimental runs from the D-optimal design

Appendix II: Plot of sample density versus monolith diameter

Appendix III: A typical TGA plot and graph of sample density vs. weight loss at $320{ }^{\circ} \mathrm{C}$ by TGA Appendix IV: Response surface models for weight loss by TGA.

Appendix V: Dependence of density on reaction time and temperature at low APTES concentration.

Appendix VI: Comparison of strength at rupture and modulus vs. density of epoxy and isocyanate crosslinked silica aerogels.

Appendix VII: Infrared spectra of typical APTES-modified, epoxy crosslinked silica aerogels. 
Appendix 1. Plot of experimental runs from the D-optimal design.

A full-factorial design to evaluate this model would contain at least 243 experiments $\left(3^{5}\right.$ experiments representing three levels each of five variables), not counting repeats. To minimize the number of experiments, however, a d-optimal experimental design strategy was used. ${ }^{1}$ To evaluate the desired model efficiently according to this type of nonclassical design, a set of experimental runs is computer-generated from the 243 candidate experiments. The computergenerated design can be described by three 3-dimensional boxes as shown in Figure S-1. Each of the three axes in each of the boxes represent three of the continuous variables: time $(t)$, APTES concentration (a) and epoxy concentration $(c)$. Each individual box represents a different level of the fourth continuous variable, temperature $(\theta)$. The discrete variable, epoxy type $e$, is represented by the three different symbols used in the boxes. Thus, each point in the boxes represents a specific experimental run in the design. The total number of unique experiments (runs) in the design is 28 . In addition, five repeats of the central sample in the design (i.e., the one that corresponds to $a=25 \%$ APTES, $e=$ tri-epoxy, $c=45 \% \mathrm{v} / \mathrm{v}$ epoxy in THF, $t=44 \mathrm{~h}$, and $\theta=50{ }^{\circ} \mathrm{C}$ ) are also required by the design in order to assess the model reliability and accuracy, assuming that the error is consistent across the whole design. In total, thirty-three aerogel samples were prepared according to Scheme 1 in random order, and were analyzed for their physical dimensions, density, surface area, porosity, strength, flexibility and dielectric properties. 

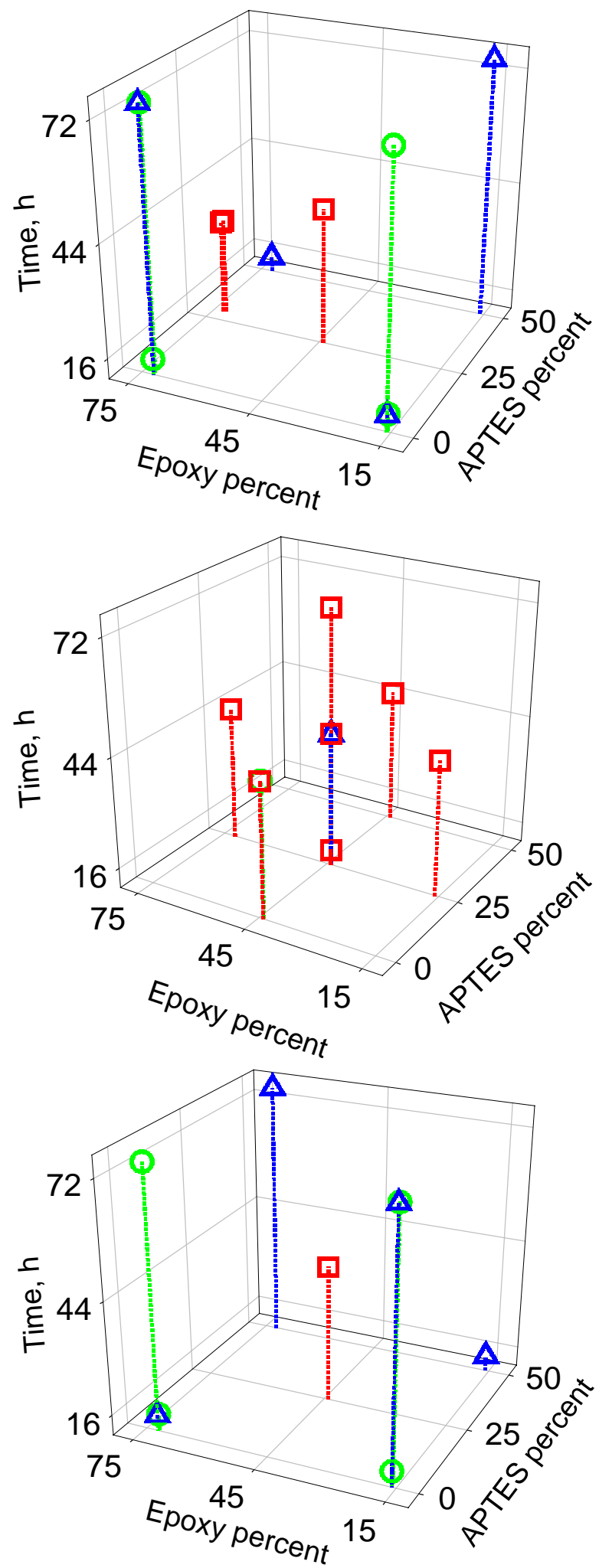

Figure S-1. Runs from the experimental design plotted in top $\left(\mathrm{t}=50 \quad{ }^{\circ} \mathrm{C}\right)$, middle $\left(\mathrm{t}=72.5{ }^{\circ} \mathrm{C}\right)$, and bottom $\left(95{ }^{\circ} \mathrm{C}\right)$. Symbols represent runs using 2-epoxy $(\mathrm{O})$, 3-epoxy $(\square)$ and 4-epoxy $(\Delta)$. 
Appendix I: $\quad$ Plot of sample density versus monolith diameter.

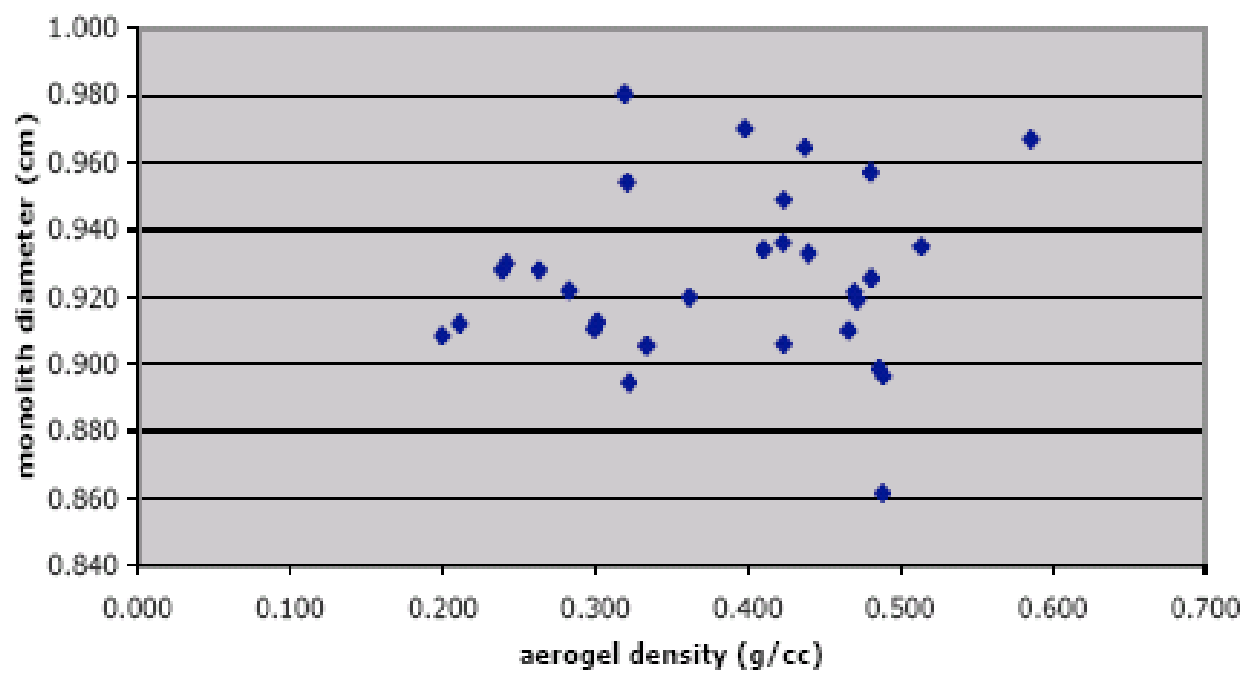

Appendix II: $\quad$ A typical TGA plot and graph of sample density vs. overall weight loss by TGA

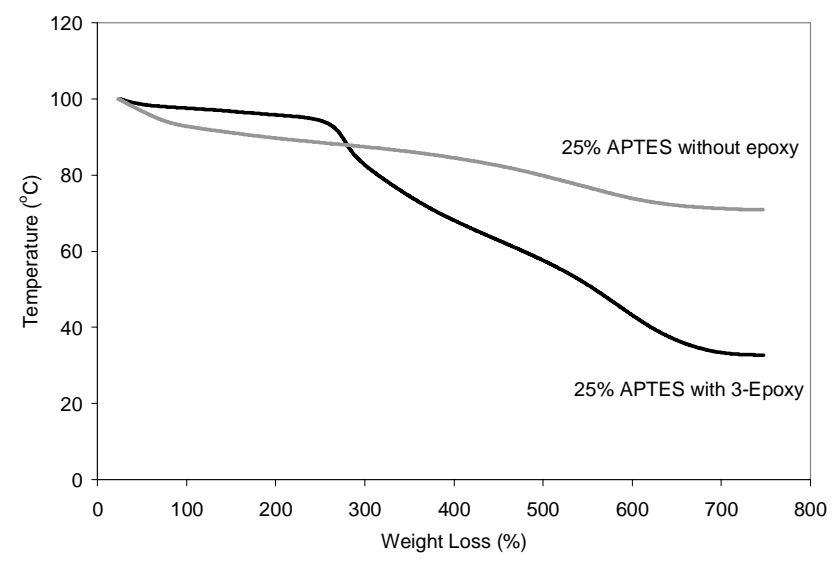

Thermogravimetric Analysis of 25\% APTES and 25\% APTES after reacting with 3-epoxy (sample No. 7). 
Appendix III: $\quad$ Response surface models for weight loss graphed $v s$. a) APTES and epoxy concentration with time and temperature held constant at the predicted optimum values for stress, and b) time and temperature with epoxy and APTES concentration held constant at the predicted optimum values for stress.

a.

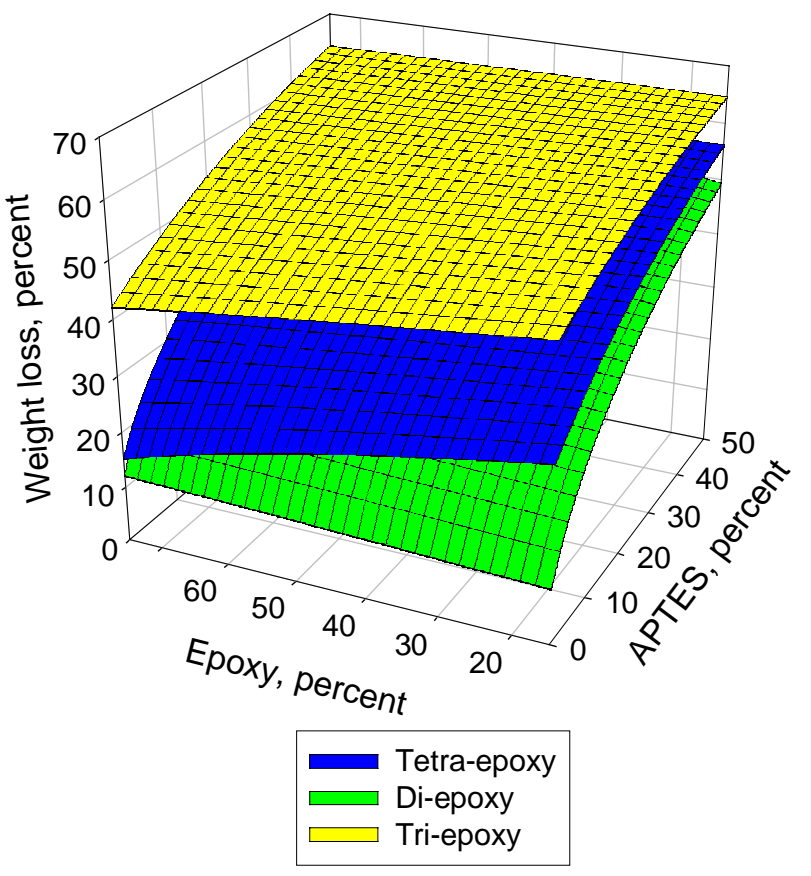

b.

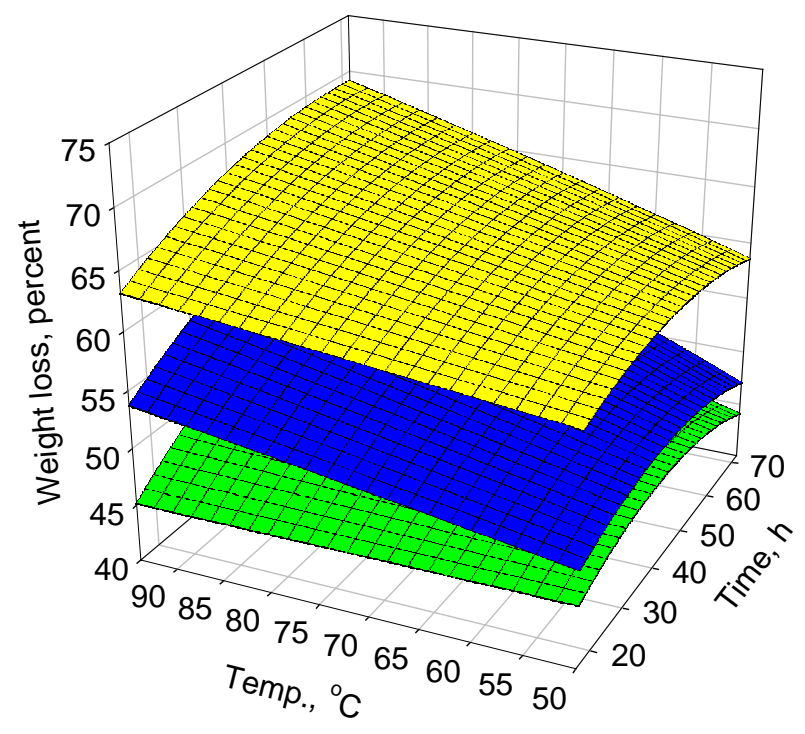


Appendix IV: $\quad$ Response surface model showing dependence of density on reaction time and temperature at $0 \%$ APTES concentration and epoxy at the optimum for stress $(54.2 \%)$.

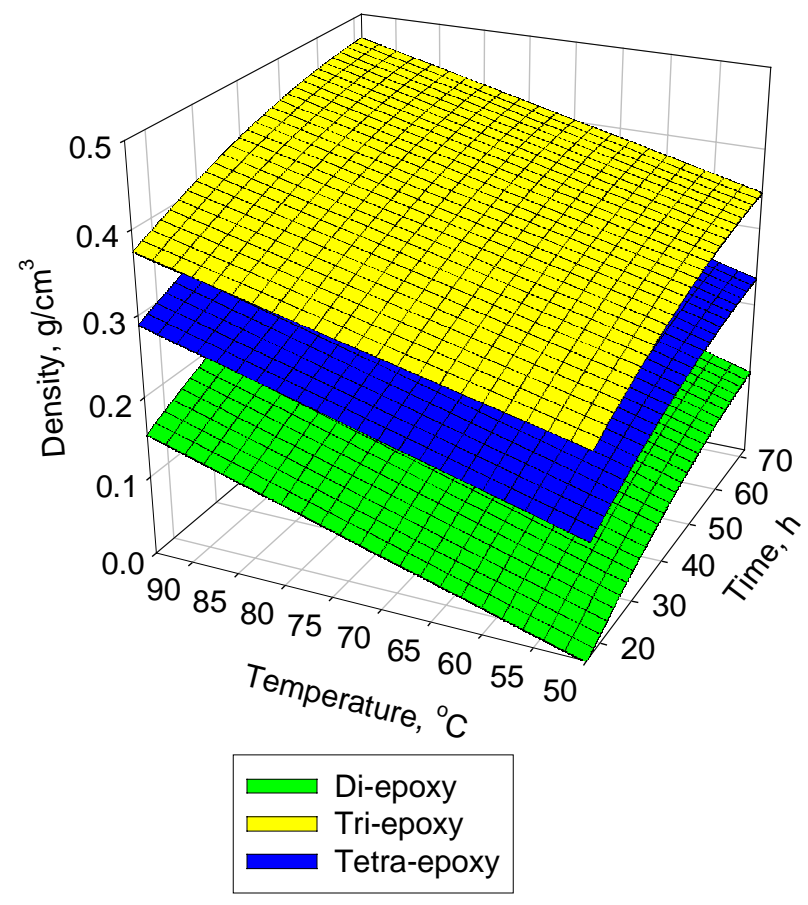


Appendix V: $\quad$ Comparison of strength at rupture and modulus vs. density of epoxy and isocyanate (Desmodur N3200 from Bayer Corporation) crosslinked silica aerogels.
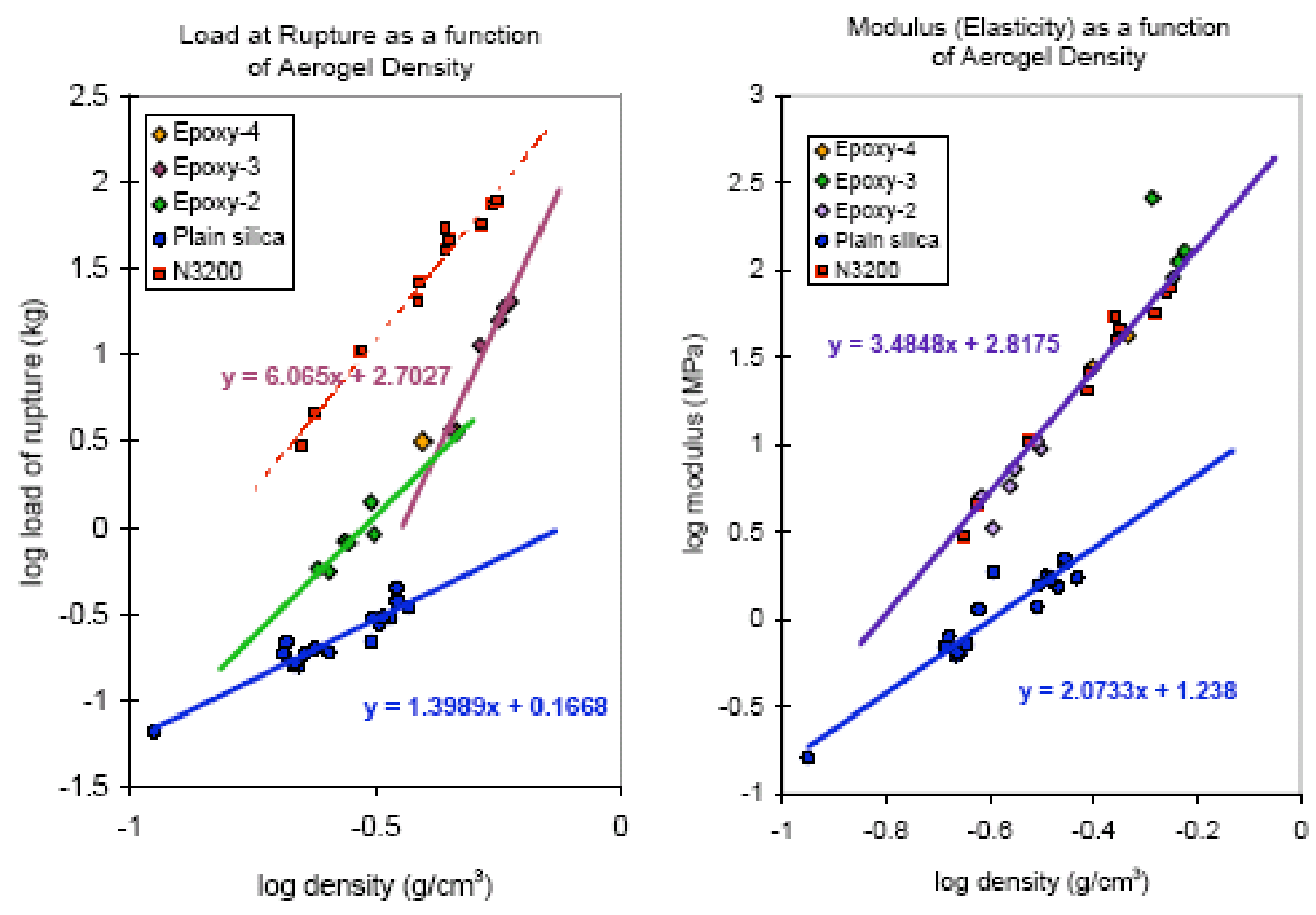
Appendix VI: $\quad$ Infrared spectra of typical APTES-modified, epoxy crosslinked silica aerogels.

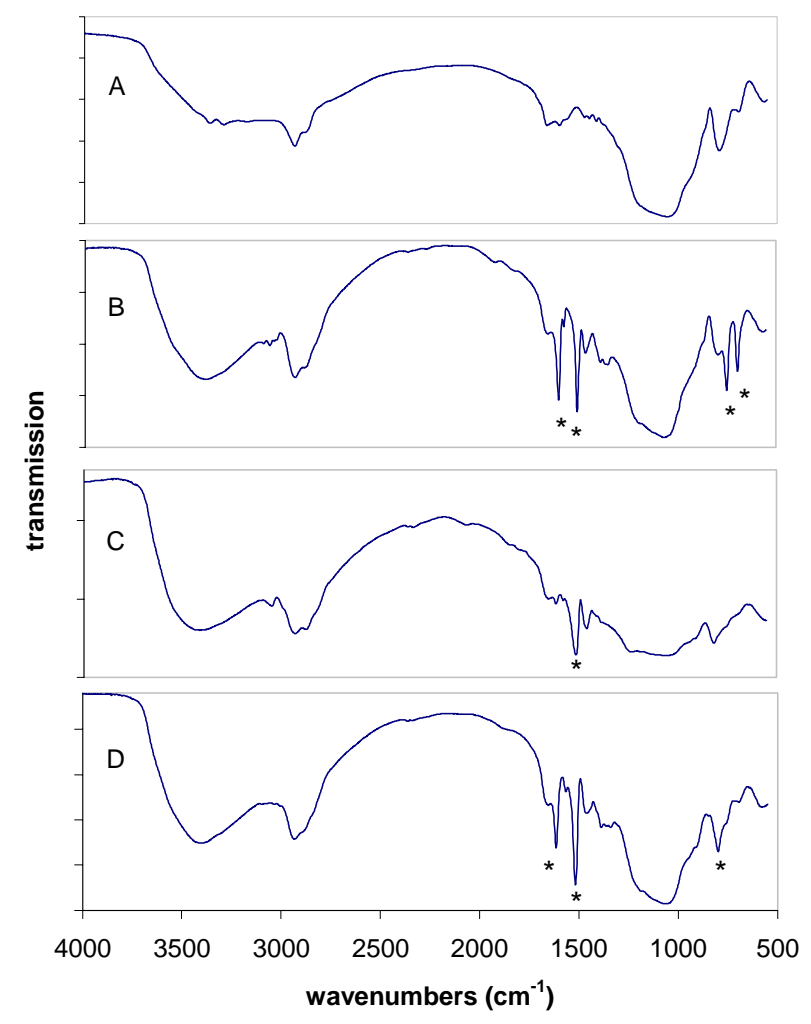

(A) 25\% APTES; (B) 25\% APTES with 2-epoxy; (C) 25\% APTES with 3-epoxy; and, (D) 25\% APTES with 4-epoxy. *: Vibrational bands attributed to the epoxies.

1. For a description of D-optimal and other statistical experimental design strategies, see Montegomery, D. C. Design and Analysis of Experiments, 1997, John Wiley and Sons, Inc., Hoboken, NJ and references therein. 